山्山FANÇAISE

$>\mathrm{DE}$

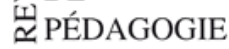

\section{Revue française de pédagogie}

Recherches en éducation

168 | juillet-septembre 2009

Enseignement et apprentissages, entre psychologie et didactiques

\title{
MERRIMaryvonne. Activité humaine et conceptualisation. Questions à Gérard Vergnaud
}

Toulouse : Presses universitaires du Mirail, 2007. - 374 p.

Marie-Jeanne Perrin-Glorian

\section{CpenEdition}

Édition électronique

URL : http://journals.openedition.org/rfp/1853

DOI : $10.4000 / \mathrm{rfp} .1853$

ISSN : 2105-2913

Éditeur

ENS Éditions

Édition imprimée

Date de publication : 1 juillet 2009

Pagination : 149-154

ISBN : 978-2-7342-1172-3

ISSN : 0556-7807

Référence électronique

Marie-Jeanne Perrin-Glorian, « MERRIMaryvonne. Activité humaine et conceptualisation. Questions à

Gérard Vergnaud », Revue française de pédagogie [En ligne], 168 | juillet-septembre 2009, mis en ligne le 07 octobre 2010, consulté le 23 septembre 2020. URL : http://journals.openedition.org/rfp/1853 ;

DOI : https://doi.org/10.4000/rfp.1853

Ce document a été généré automatiquement le 23 septembre 2020

(c) tous droits réservés 


\section{MERRIMaryvonne. Activité humaine et conceptualisation. Questions à Gérard Vergnaud}

Toulouse : Presses universitaires du Mirail, 2007. - 374 p.

Marie-Jeanne Perrin-Glorian

\section{RÉFÉRENCE}

MERRIMaryvonne. Activité humaine et conceptualisation. Questions à Gérard Vergnaud.

Toulouse : Presses universitaires du Mirail, 2007. - 374 p.

Cet ouvrage est issu du colloque qui s'est tenu à Paris du 28 au 31 janvier 2004 en l'honneur de Gérard Vergnaud. Le livre de 375 pages (dont 17 pages regroupant les références bibliographiques de tous les textes) est complété par un CD-ROM, ce qui donne un ouvrage de 826 pages composé de multiples contributions présentant, utilisant, questionnant, complétant l'œuvre de Vergnaud. Il n'est pas facile d'en rendre compte vu la quantité et la diversité des textes qu'il contient, à la frontière de plusieurs domaines de recherche. La didactique des mathématiques (où mes propres travaux s'inscrivent) y est présente ainsi que des didactiques d'autres disciplines (les sciences notamment), à côté de la didactique professionnelle, de la didactique comparée et de la psychologie bien sûr puisque c'est au carrefour de ces disciplines que se situe l'œuvre de Vergnaud, qui a contribué de façon décisive à la naissance de plusieurs d'entre elles. Le point de vue que je donnerai sera donc très partiel, puisque je suis loin de connaître suffisamment tous les cadres théoriques et toutes les problématiques invoquées dans cet ouvrage. Je demande l'indulgence des auteurs dont j'aurais mal saisi le propos. Cette note est trop courte pour rendre compte de chacune des interventions. Je ferai une présentation d'ensemble puis j'essaierai de tirer quelques fils que j'invite le lecteur à suivre tout en étant sûre qu'il aura envie d'en tirer d'autres. En effet, l'ouvrage ne se lit pas de façon linéaire: il procède par touches. Le lecteur a à faire un travail de 
recomposition et de confrontation des différentes contributions; il y trouvera sans doute des choses différentes suivant sa culture de départ et ce qu'il vient y chercher. Pour le lecteur averti qui vient avec des questions sur les travaux de Vergnaud et ce qu'ils peuvent lui apporter, c'est une référence et une occasion d'enrichir ou d'assurer ses propres cadres théoriques. Pour le lecteur plus novice qui s'intéresse aux questions concernant la conceptualisation, ses liens avec les didactiques, le développement de compétences professionnelles, cet ouvrage donne des entrées multiples dans l'œuvre de Vergnaud et la manière dont elle se situe par rapport à d'autres courants de pensée et d'autres cadres théoriques en psychologie et en didactique. Il y trouvera aussi des clés pour d'autres travaux, notamment en psychologie ou en didactique professionnelle.

2 Concernant la présentation générale, le livre est organisé en quatre parties encadrées par deux textes de Vergnaud: dans le premier, "Héritages ", il livre ses sources et donne les clés de la théorie des champs conceptuels (notée TCC dans la suite) et de sa genèse historique; dans le dernier, "Réponse ", il répond à certaines des questions soulevées, en les organisant autour de trois nœuds du questionnement: «la conceptualisation ", « la référence ", « la vérité et la pertinence ». Il revient aussi sur sa thèse, peu connue et commentée dans l'ouvrage par J.-F. Richard, où il établissait un classement des situations selon deux axes, l'un caractérisant la détermination de la situation (nécessaire, régulière, aléatoire), l'autre le degré de liberté du sujet dans son action sur la situation (productive, passive, interactive), le sujet étant le seul agent du changement dans la situation productive. Il émettait l'hypothèse que la rationalité se construisait d'abord dans les situations nécessaires, avec un privilège pour celles qui étaient de plus productives. Le CD-ROM s'ouvre aussi sur un texte de Vergnaud intitulé "Perspectives" dans lequel il complète ses réponses aux intervenants et reconnaît dans les contributions des perspectives pour un approfondissement des concepts de schème et d'invariants opératoires, notamment en didactique professionnelle, comme par exemple un élargissement «à des aspects de l'activité non directement adressés à des classes de situations étroitement définies ».

3 La première partie du livre, intitulée "Rien de plus pratique qu'une bonne théorie, mais... ", d'une part aide à voir l'ancrage de la théorie des champs conceptuels dans l'œuvre de Piaget et en même temps la manière dont elle s'en démarque (notamment dans les textes de Gérard Sensevy, Jean Brun et de Pierre Pastré), d'autre part présente les apports de la TCC en didactique des mathématiques (Gérard Sensevy, Guy Brousseau, Jean-Baptiste Lagrange, Jean Brun, Colette Laborde, Rudolf Straßer) ou en didactique professionnelle, y compris pour la profession enseignante (Pierre Pastré, Pierre Rabardel, Roland Goigoux). On y voit comment la TCC, et en particulier les notions de schème et de concept avec leurs composantes, notamment les invariants opératoires et les situations, peuvent s'articuler avec d'autres cadres théoriques complémentaires comme la théorie des situations didactiques ou se prolonger en se spécifiant à de nouveaux contextes comme dans l'approche instrumentale étudiant l'appropriation par les enseignants et les élèves des artefacts issus principalement des nouvelles technologies.

4 Dans la deuxième partie "Questions, alternatives et compléments ", huit chercheurs (Jean-François Richard, Jean-Paul Bronckart, Michel Recopé, Janine Rogalski, Yves Clot, Maria-Luisa Schubauer-Leoni, Patrick Mayen, Jean-Louis Martinand) discutent certains choix théoriques de l'œuvre de Vergnaud, en les confrontant éventuellement à d'autres 
points de vue qu'ils utilisent ou qu'ils ont eux-mêmes développés. Cette partie est une nouvelle occasion de revenir sur les principaux concepts de la TCC, et en particulier les notions de schème et d'invariants opératoires et de les situer par rapport à ceux qu'avait introduits Piaget. C'est souvent aussi l'occasion pour les divers auteurs d'expliquer leur cheminement, de préciser leurs propres options théoriques, et de les situer par rapport aux travaux de Vergnaud.

5 Le questionnement théorique de l'œuvre de Vergnaud, qui commence en fait dès la première partie, se poursuit dans la troisième, "Plus loin sur la conceptualisation ", avec six textes qui situent les travaux de Vergnaud par rapport à d'autres cadres théoriques, en proposant éventuellement des moyens de les y articuler. Alain Trognon et Viviane Durand-Guerrier discutent la place de la logique dans la théorie des champs conceptuels; Marianne Lacomblez compare les apports respectifs de Vergnaud et Faverge en psychologie du travail ; Alain Mercier relève une extension notable de la notion de schème par le fait d'y inclure des représentations externes au sujet, et il propose de s'engager dans l'étude des liens entre notations et invariants opératoires; Terezinha Nunes, en collaboration avec cinq autres collègues, propose un prolongement au cas des fractions des travaux de Vergnaud sur l'impact des situations sur le développement des opérations sur les nombres entiers; Françoise DuquesneBelfais et Claire Meljac discutent, en s'appuyant sur quatre exemples, les importations récentes en psychologie de références issues d'autres champs disciplinaires.

6 La quatrième partie, "Individu et société ", regroupe cinq contributions de chercheurs engagés dans des réalisations didactiques de grande envergure ou dans l'étude de dimensions sociales de la formation. Boero met en relation la TCC et ses propres travaux à propos de la contribution des expériences extra-scolaires des élèves à la construction de concepts mathématiques dans le cadre scolaire. Jorge Tarcisio da Rocha Falcao pose la question des rapports entre conceptualisation en actes et conceptualisation explicite : «Que savent ceux qui

savent comment mais ne savent pas pourquoi? " Jean-Claude Coulet témoigne, à partir de l'exemple des directeurs d'écoles paramédicales, de la pertinence de la TCC pour construire des référentiels de compétences professionnelles. Il aborde aussi la question de l'évolution des schèmes au cours de la vie professionnelle, par la prise en compte des effets en retour suite à la mise en œuvre du schème; il envisage ainsi différentes formes de régulation de l'activité qui amènent une évolution plus ou moins forte des schèmes. Esther Pillar Grossi témoigne de l'apport de la TCC dans la mise en place de structures d'alphabétisation au Brésil par un groupe de recherche qui fonctionne depuis 34 ans. Christine Dollo et Samuel Johsua s'intéressent à la construction des compétences dans des lieux autres que l'école ou le milieu professionnel, par exemple les centres de formation des partis politiques ou des syndicats, mais aussi des systèmes plus « silencieux » où l'apprentissage se fait davantage par imprégnation, comme dans la famille. Ils suggèrent que l'étude de la conceptualisation dans ce tiers secteur, qui a certains points communs avec la psychologie du travail mais s'en démarque par d'autres côtés, pourrait être utile aussi pour la didactique.

7 Nous avons choisi de rendre compte maintenant de trois fils de discussion qui traversent l'ouvrage : autour des concepts de la théorie des champs conceptuels et en particulier $\mathrm{du}$ couple schème/situation et de leurs composantes; autour de l'articulation entre les théories de Piaget et de Vygotski; autour de la didactique professionnelle. 
Jean Brun le souligne (p. 68), Vergnaud lui-même le répète à plusieurs reprises : «le couple schème-situation est la clé de coûte de la psychologie cognitive et de la théorie de l'activité » ou tout au moins de la TCC, puisque « la connaissance étant adaptation, ce sont les schèmes qui s'adaptent et ils s'adaptent à des situations ». Mais la notion même de situation n'est pas transparente. Brousseau (p. 49-62), en comparant la notion de situation en TCC aux deux sens de situation dans la théorie des situations (situation mathématique et situation didactique), amène un éclairage en même temps sur la théorie des situations et sur la théorie des champs conceptuels. Selon lui, en TCC, les situations sont un moyen de rendre compte de la partie organisée de la réalité qui se manifeste à propos d'un concept. Du point de vue de la théorie des situations, il s'agit alors d'une situation sans intervention didactique, où le sujet ne perçoit pas d'intention didactique correspondant au niveau de la situation "objective ", c'est-à-dire le milieu sur lequel agit le sujet. De plus, il remarque que Vergnaud utilise de façon presque équivalente les termes "tâche » et "situation » alors que, de son point de vue, la différence est très nette: " une tâche est ce que le sujet doit faire en réponse à une situation ». Brousseau considère qu'on ne peut pas décrire les conditions de l'action du sujet, la situation, par l'action elle-même, la tâche. Pour Boero (p. 287) la notion de situation de référence, qu'il met en relation avec la conceptualisation, ne peut pas non plus se résumer à une tâche puisque, suivant le contexte éducationnel, la même tâche peut engendrer ou non des productions significatives de la part des élèves. Le sens du concept se trouve pour lui «dans l'activité accomplie par le sujet à propos d'une tâche bien choisie dans un contexte éducationnel approprié » et "l'activité met en jeu des choses qui sont à l'extérieur de la tâche et même de son contexte immédiat ». Brousseau discute aussi les classements de situations dans les deux théories et le problème du comportement de la correspondance connaissance/situation dans les évolutions des situations ou des connaissances. Dans sa réponse, Vergnaud précise à ce sujet (p. 346) : « les six relations de base que j'ai dégagées [pour les structures additives] ont à mes yeux la même fonction pour l'ensemble des problèmes d'addition et de soustraction, que celle d'une base de vecteurs pour un espace vectoriel. Chacune de ces relations est irréductible aux autres; mais, en se combinant, elles permettent d'engendrer un immense réseau relationnel, l'ensemble du champ conceptuel des structures additives.» Il ajoute (p.345) que la référence des situations peut être cherchée aussi bien dans la vie ordinaire que dans l'épistémologie et l'histoire.

Plusieurs auteurs discutent les apports de Vergnaud à la notion de schème de Piaget. Par exemple, J.-F. Richard considère que c'est surtout la notion de théorème en acte que Vergnaud a ajoutée au schème piagétien. Pour Mercier, un changement profond de la notion de schème et une manière de l'étendre bien au-delà de son domaine initial vient de l'inclusion des signifiants : «les schèmes ont aussi une dimension externe au sujet et cette dimension porte certains des invariants opératoires (p. 246) ». Sensevy (p. 45-46) souligne aussi que les signes, en tant que composantes de la construction des schèmes et des concepts sont un des moyens de la prise de distance par rapport aux situations. De son côté, Bronckart (p. 133) considère qu'une modification substantielle (et presque contradictoire) apportée par Vergnaud à la notion de schème de Piaget est la radicalisation qui lui fait considérer que le schème est accessible au sujet et constitue l'organisateur de sa pensée consciente. En effet, selon lui, le schème chez Piaget, d'une part relève d'une lecture externe par le chercheur du fonctionnement sensori-moteur ou des opérations logico-mathématiques d'un sujet et non du fonctionnement conscient de la pensée du sujet observé et d'autre part, Piaget considère que le 
fonctionnement causal des lois physiques s'applique aussi aux schèmes, en tant que "régularités observables du dehors ", mais qu'il ne peut qualifier les mécanismes de la pensée consciente qui se caractérisent par des enchaînements d'implications signifiantes, régis par des règles non strictement nécessaires. Une autre modification substantielle de la notion de schème est selon lui (p.134) l'ouverture constituée par l'articulation des schèmes aux situations dans lesquelles ils se déploient. Il trouve d'ailleurs que cette ouverture ne va pas assez loin et qu'elle aurait pu amener Vergnaud à prendre en compte les dimensions structurantes et signifiantes (pour la construction des schèmes) des préconstruits socio-historiques auxquels les individus sont confrontés. Mayen, quant à lui, analyse les relations entre schèmes et médiation en s'appuyant sur Bruner. Il montre que la TCC permet d'analyser l'action de tutelle en lien avec les composantes du schème et avec les composantes de la situation sur lesquelles l'enseignant peut agir (buts, état des variables concernant l'objet de l'action ou les conditions de l'action, ressources matérielles, symboliques ou humaines pour le faire ${ }^{1}$ ). En même temps, il pointe les limites de ses apports dans la compréhension des conditions et des processus d'apprentissage et de développement en ce qui concerne les autres et la culture, dans la mesure où elle limite le rôle de la médiation à une sorte d'accompagnement du développement. « La mise en relation, pour l'action enseignante, des fonctions d'étayage et du schème dans sa définition analytique concrétise très clairement comment le concept de schème peut contribuer à répondre à "ce qui manque le plus" à la problématique de l'apprentissage et du développement initiée par Vygotski. Réciproquement, elle montre aussi combien la prise en compte des médiations d'autrui peut apporter à la théorie des schèmes. " (p. 194) Il propose de définir deux versions de la tutelle : la tutelle "forte » qui correspond à la création de zones de développement et la tutelle "faible» qui correspond seulement à l'actualisation de zones de développement. Dans la version forte, l'apprenant «est conduit à rechercher et à retrouver la situation qui correspond à l'orientation qui lui a été donnée par celui qui l'aide » (p. 197). Il insiste aussi sur le rôle du langage comme source du développement. Outre les invariants opératoires, beaucoup d'auteurs indiquent aussi le lien entre schèmes et situations comme un des éléments qui changent le plus profondément la notion de schème et certains étendent d'ailleurs la notion de schème dans le sens souhaité par Bronckart. Ainsi, pour M.-L. SchubauerLeoni (p. 190), «si les schèmes ont bien une fonction d'organisateurs de l'activité pour des classes de situations données, ils sont pris dans des dispositions produites (culturellement) dans le rapport agent-situation sous couvert d'une institution donnée. Ceci signifie qu'il convient d'attribuer aux schèmes une origine interpsychologique dont la conversion intrapsychologique est réglée contractuellement. Les échanges langagiers, et plus profondément les signes (arbitraires et engendrés culturellement, socio-historiquement et conventionnellement) s'avèrent constitutifs de la pensée du sujet, y compris dans les moments où il pense et agit "tout seul" ».

Dans sa réponse (p. 341), Vergnaud souligne que c'est un point de vue développemental et pragmatique qui permet de comprendre ce qu'est un concept et que c'est ce qui l'a conduit à considérer le couple situation/schème comme le couple fondamental de la psychologie développementale. Il ajoute qu'il n'y a pas pour lui de contradiction entre construit personnel et construit culturel, d'autant plus que les construits culturels sont aussi le plus souvent au départ des construits personnels. Il insiste aussi sur la nécessité de ne pas séparer la forme opératoire et la forme prédicative de la connaissance et donne l'exemple, pour la proportionnalité et les structures multiplicatives, de 
graphismes qui peuvent être fournis aux élèves comme aide au raisonnement. Dans son texte final, il revient (p. 384) sur la notion de script-algorithme qu'il avait introduite «justement pour indiquer l'intrication de la progression de l'activité de pensée avec la progression des formes symboliques utilisées. » Il dit aussi (p. 384) que "l'idée de rétroaction sur l'activité d'un certain résultat temporairement accepté, est en fait cruciale pour la compréhension du concept de schème, puisque celui-ci n'est pas un moyen d'engendrer l'activité après une seule prise d'information initiale, qui serait une sorte de déclencheur, mais un moyen d'engendrer l'activité au fur et à mesure, avec ce que cela comporte d'actions, de nouvelles prises d'information, de contrôles, d'inférences, et d'élaboration de sous-buts ad hoc. »

11 La discussion autour de la notion de schème contient en arrière-plan la question de l'articulation entre les théories de Piaget et de Vygotski. Bronckart aborde directement la question de la compatibilité de ces théories. Selon lui, si l'on prend au sérieux les positionnements philosophiques orientant le travail des psychologues, comme le recommande Vergnaud lui-même, on ne peut considérer que les approches de Piaget et de Vygotski sont complémentaires parce qu'elles reposent sur des positions philosophiques radicalement opposées. En effet l'œuvre de Piaget s'inscrit dans le prolongement de celle de Kant, amendée notamment par la considération de la construction progressive des capacités cognitives au cours de l'ontogenèse, capacités qui s'inscrivent dans le développement continu des espèces. Cette position conduit à une sous-estimation du langage, de la culture et de la formation. Le sémiotique et le social sont secondaires, produits de la mise en œuvre des capacités cognitives de traitement du monde. Pour Vygotski, héritier de Spinoza, Marx et Engels, l'ordre est inversé : l'objet premier est la praxis collective et l'intériorisation des structures et significations sociales transforme radicalement le psychisme hérité et donne naissance aux capacités de pensée consciente. Dans son texte, le plus long de l'ouvrage (21 p.), Bronckart procède à une lecture critique de l'œuvre de Vergnaud à laquelle il porte deux critiques principales (p. 135-137) : la première est de s'appuyer sur l'agir sans le définir et en particulier sans distinguer «activité » qui peut s'appliquer à toute organisation collective orientée par une finalité de survie ou visant un objet déterminé (par exemple la danse des abeilles) et « action » qui s'applique « à l'agir collectif en tant qu'il est articulé à des buts que les agents concernés se représentent ou dont ils ont conscience ». La deuxième est la place du langage : il considère pour sa part que c'est l'appropriation et l'intériorisation des signes langagiers qui sont constitutives des unités de pensée, parce que c'est dans le cadre de formes socio-langagières particulières et arbitraires que s'organisent les représentations humaines. De plus, pour lui, c'est l'appropriation et l'intériorisation des structures langagières qui sont à l'origine de l'organisation de ces unités en un système de pensée consciente parce que les implications de significations ne peuvent dériver de la logique de nécessité des enchaînements causaux. Cependant Bronckart, qui adhère globalement à la position de Vygotski, reconnaît que la théorie de celui-ci doit être complétée : il lui manque peutêtre une conception générale des opérations cognitives et des schèmes, comme l'affirme Vergnaud, et une conception de la personne et de son statut qu'il tente de réintégrer à la fin de son texte. Il propose ainsi (p.139-141) d'intégrer les apports de Piaget au schéma développemental vygotskien. La pensée consciente se construit pour lui dans l'interaction avec des préconstruits socio-sémiotico-culturels élaborés par les générations précédentes; elle porte donc les traces d'un certain marquage social, à la fois d'ordre sémiotique et d'ordre actionnel. Mais «l'influence sociale n'est jamais 
déterminisme radical parce que chaque enfant est confronté à des préconstruits sociaux dans des contextes de médiation formative qui peuvent présenter des caractéristiques très différentes [...] parce que ces expériences s'accumulent selon une temporalité qui est toujours particulière, et parce que les conditions des expériences acquises engendrent, à terme, un cadre d'accueil personnel exerçant une influence sur les interactions formatives ultérieures. L'enfant construit donc la structure psychologique constitutive de sa personne dans le cadre d'une micro-histoire expérientielle singulière, qui relève d'un régime fondamentalement distinct de celui de l'histoire sociale » (p. 140).

La didactique professionnelle quant à elle s'est appuyée sur la notion de schème mais l'a prolongée en y intégrant clairement une dimension sociale et même, pourrait-on dire, institutionnelle, ainsi que le rôle du langage. Cette extension a demandé des adaptations. Ainsi Pastré (p. 84-86), tout en décrivant la filiation entre la TCC et la didactique professionnelle, souligne deux grandes différences: d'une part l'organisation du champ conceptuel se fait autour de la cohérence du savoir alors que celle des champs professionnels se fait de façon empirique autour des situations; d'autre part la dimension matérielle des transformations est essentielle dans les champs professionnels alors qu'elle est secondaire pour les champs conceptuels. Il analyse la construction d'un champ conceptuel comme la transformation de la variété des situations en une variation de problèmes rationnellement organisés ${ }^{2}$, démarche qu'on retrouve en didactique professionnelle : on extrait la structure conceptuelle de la situation professionnelle en identifiant des concepts organisateurs, des moyens de catégoriser. L'organisation du champ conceptuel se fait autour du couple concept/ problèmes plus qu'autour de savoirs et procède par rectifications. Rabardel (p. 88) insiste aussi sur l'altérité entre didactique disciplinaire et didactique professionnelle parce que, dans le champ professionnel, ce qui est premier, c'est l'action efficace. La didactique professionnelle a dû renverser la centration de la didactique disciplinaire sur des connaissances et des savoirs pour se situer dans une perspective épistémologique caractérisée par deux dimensions principales : celle des capacités du sujet à agir sur des situations et celle des ressources que le sujet construit, élabore, s'approprie... pour assurer ce pouvoir de faire. Cela l'a amené à distinguer, avec R. Samurçay, les activités productives et les activités constructives et à se recentrer sur le sujet, mais un sujet qui évolue. Un autre aspect spécifique de la didactique professionnelle est la dialectique entre appropriation et mise en patrimoine des savoirs : on n'a pas seulement appropriation mais aussi création, qui ajoute du nouveau à disposition d'une communauté et participe au renouvellement de sa culture. Janine Rogalski (p.168) ajoute que la marque des concepts disciplinaires, en mathématiques par exemple, "est leur polyvalence comme outils utilisables dans une variété de situations dans lesquelles ce champ conceptuel va opérer»; en revanche dans le domaine du travail, «les champs de situations sont relativement circonscrits » alors que les champs disciplinaires qui nourrissent ou justifient la construction des procédures sont multiples. Elle discute aussi la notion de schème en lien avec le développement des compétences. Elle remarque notamment que, s'il est assez facile de repérer des schèmes locaux, «les invariants de plus haut niveau de l'organisation de l'activité sont moins aisés à identifier, si on ne confond pas l'existence de schèmes partagés, repérés dans une situation par des invariants entre sujets, et l'existence des "mêmes" schèmes individuels dans une classe de situations. " Yves Clot développe justement la notion de schème collectif. Pour lui, du point de vue de la psychologie du travail, le problème des rapports entre schèmes individuels et schèmes collectifs se 
pose de façon essentielle et on est amené à regarder la question des organisateurs invariants de l'action en partant du collectif pour aller vers le sujet et non l'inverse. En effet, en situation professionnelle, les organisateurs de l'action sont «des patrons sociaux, des manières de prendre les choses, les gens, encouragés ou inhibés dans un milieu de travail donné » (p. 181). De plus ce ne sont pas des figures imposées mais plutôt des systèmes de variantes que chacun doit recréer pour se l'approprier: la formation d'un schème personnel se fait par création d'un style personnel à partir du genre professionnel. Pour lui, les invariants sont le produit de l'expérience répétée des variations dont la source est à chercher du côté du contact des sujets avec le monde extérieur. C'est à l'aide d'instruments génériques, revus et corrigés par lui que le sujet se heurte à l'opposition des objets réels. Les invariants sont en position de ressource. Ainsi, Clot est reconnaissant à Vergnaud de l'avoir aidé à comprendre combien, au plan conceptuel, le schème explique l'activité, même si, selon lui, dans la vie, et selon la perspective vygotskienne et wallonnienne qui lui sert de référence, « c'est l'activité qui s'explique avec le schème, aux deux sens du terme » (p. 183). Goigoux (p. 91-102), quant à lui, explique comment, pour étudier les pratiques professionnelles des enseignants et leur développement, il utilise complémentairement les concepts de schème professionnel, en mettant au centre le couple schème/situation (le schème est une forme organisée et stabilisée de l'activité d'enseignement pour une classe de situations professionnelles qui doit être identifiée) et de genre professionnel comme répertoire collectif de schèmes socialement et historiquement construits. La notion de schème est bien adaptée pour rendre compte de la manière dont l'enseignant est capable de prendre rapidement des décisions en situation, par exemple face à des difficultés d'élèves. Goigoux fait d'ailleurs l'hypothèse que la qualité de ces ajustements didactiques est l'un des fondements de l'expertise professionnelle des enseignants. Selon lui Clot «prolonge la réflexion de Vergnaud dans le champ professionnel en l'enrichissant d'une dimension collective, historiquement et socialement située, pour étudier la constitution des répertoires de schèmes " et la notion de genre "aide à mieux théoriser les ressources et les contraintes qui pèsent sur l'activité enseignante : il dit ce qu'il est bon de faire et de ne pas faire [...] en même temps qu'il soutient l'action, il la contraint".

13 Bien sûr, la discussion sur tous ces points devrait être complétée par les apports du CDROM joint à l'ouvrage. Le premier thème, coordonné par Maryvonne Merri, apporte justement 7 contributions sur "situation et schème"; le second, coordonné par Isabelle Vinatier, amène 8 nouvelles contributions sur «champ conceptuel, champ professionnel »; le troisième, coordonné par Jeanne Guiet-Silvain, rassemble 8 textes sur "processus individuels, collectifs et culturels dans la conceptualisation » et le quatrième, coordonné par François Cauvin, comprend 8 autres textes sur " connaissances implicites, explicites et perception ». D'ailleurs, dans son texte final, au début du CD-ROM, Vergnaud fait une synthèse de l'ensemble de l'ouvrage et apporte des réponses aussi bien aux contributions du livre qu'à celles du CD-ROM. Bonne lecture! 


\section{NOTES}

1. On peut reconnaître ici les règles du jeu et le milieu de la théorie des situations.

2. Et Vergnaud trouve cette idée particulièrement féconde (texte « Perspectives », p. 381).

\section{AUTEURS}

MARIE-JEANNE PERRIN-GLORIAN

Laboratoire de didactique André-Revuz, université d'Artois 